\title{
Acute appendicitis complicated by mass formation occurring simultaneously with serologically proven dengue fever: a case report
}

Manouri P Senanayake $e^{1,2^{*}}$ and Malik Samarasinghe $e^{2,3}$

\begin{abstract}
Introduction: Acute abdomen and acute appendicitis are unusual clinical presentations that occur in dengue infection-caused illness. Lymphoid hyperplasia and mesenteric adenitis are possible explanations, although vasculitis in the pathology of dengue infection has not been reported. Authors of previous case reports have described mimicking of acute appendicitis discovered upon surgical treatment. Dengue virus has not been proven to cause acute appendicitis.

Case presentation: We report a case of an 8-year-old Sinhalese boy who developed acute appendicitis during the acute phase of serologically confirmed dengue fever. Although abdominal pain, vomiting and right-sided tenderness were present at the time of admission, a diagnosis of acute appendicitis was considered only 18 hours later, when abdominal guarding and a well-defined mass in the right iliac fossa were detected clinically and ultrasonographically. Conservative management with intravenous antibiotics was successful.

Conclusion: In areas where dengue is endemic, awareness of dengue viral infection as a non-surgical cause of acute abdomen, as well as its ability to mimic acute appendicitis, is important because unnecessary surgery-related morbidity can be decreased. However, delaying or missing the diagnosis of acute appendicitis can result in serious complications. This message is particularly relevant to clinicians, especially pediatricians and surgeons, who encounter large numbers of patients during dengue epidemics and run the risk of missing the diagnosis of acute appendicitis. Likewise, delaying or missing the diagnosis of dengue hemorrhagic fever can lead to dengue shock syndrome and even death. This case highlights the need for careful evaluation of each patient who presents with acute abdomen and dengue infection.
\end{abstract}

Keywords: Acute appendicitis, Dengue fever

\section{Introduction}

Dengue infection is known to mimic acute appendicitis. The resultant diagnostic confusion has been highlighted in several case series, and dengue is now a recognized nonsurgical cause of acute abdomen [1-5]. In our case report, we describe the rare occurrence of both dengue infection and acute appendicitis in a child living in a dengueendemic area. This case highlights the need for careful evaluation, a message which is particularly relevant in

\footnotetext{
* Correspondence: manouri.senanayake@gmail.com

'Department of Pediatrics, Faculty of Medicine, University of Colombo, Kynsey Road, Colombo, Sri Lanka

${ }^{2}$ Lady Ridgeway Hospital for Children, Colombo, Sri Lanka

Full list of author information is available at the end of the article
}

dengue epidemics, when the possibility of delays in the diagnosis of acute appendicitis is high.

\section{Case presentation}

During a dengue epidemic in Sri Lanka, an 8-year-old Sinhalese boy presented to our children's hospital in Colombo on the fourth day of high fever that had responded poorly to paracetamol. Severe myalgia and anorexia were present from the onset of illness. Abdominal pain and vomiting had set in on the second day of symptomatic infection and abdominal pain continued for six days after hospital admission. His examination showed that he had a flushed appearance and was febrile

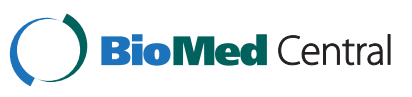

(c) 2014 Senanayake and Samarasinghe; licensee BioMed Central Ltd. This is an Open Access article distributed under the terms of the Creative Commons Attribution License (http://creativecommons.org/licenses/by/2.0), which permits unrestricted use, distribution, and reproduction in any medium, provided the original work is properly credited. The Creative Commons Public Domain Dedication waiver (http://creativecommons.org/publicdomain/zero/1.0/) applies to the data made available in this article, unless otherwise stated. 
and moderately dehydrated. His Hess test was positive. A tender hepatomegaly of $2 \mathrm{~cm}$ and tenderness in the lower right abdomen were present. The patient's spleen was not palpable. Laboratory tests revealed leukopenia $\left(4 \times 10^{9} / \mathrm{L}\right.$ white blood cell count), neutrophil leukocytosis (92\%), thrombocytopenia $(86 \times 109 / \mathrm{L})$, raised hematocrit level (42\%), and mildly elevated liver enzymes (aspartate aminotransferase, 101IU/L; alanine aminotransferase, 48IU/L). A clinical diagnosis of dengue fever was made. The boy's dehydration was corrected with intravenous fluid therapy. The presence of immunoglobulin G (IgG) and IgM dengue antibodies provided serological confirmation of the diagnosis.

The patient was monitored for evidence of dengue hemorrhagic fever (DHF) and dengue shock syndrome. Plasma leakage and circulatory instability were absent. Eighteen hours after the boy's admission, his abdominal tenderness became more marked. We observed localized abdominal guarding and felt a well-defined mass in the right iliac fossa. A tender mass in the right iliac fossa with surrounding intraperitoneal fluid suggestive of an appendicular mass was confirmed by ultrasonography. The patient's serum C-reactive protein (CRP) level was $44.0 \mathrm{mg} / \mathrm{L}$ (normal, $<8 \mathrm{mg} / \mathrm{L}$ ). His urine culture was sterile. Concomitant acute appendicitis with dengue fever was diagnosed clinically and on the basis of ultrasonography.

The boy's illness was managed conservatively with intravenous cefotaxime and metronidazole. The patient's leukopenia and thrombocytopenia resolved on the sixth day of illness, and we observed the characteristic rash that appears upon recovery from dengue. However, his fever, neutrophil leukocytosis, and raised CRP level persisted. We attributed these symptoms to appendicitis and mass formation. We administered intravenous antibiotics, which led to a positive response, and the patient was free of pain and gastrointestinal symptoms when he was discharged to home. An interval appendectomy was planned. His hospital stay of 13 days exceeded the average length of stay for dengue fever.

\section{Discussion and conclusion}

Dengue is an important viral infection in the world today. Lymphoid hyperplasia and mesenteric adenitis may be possible underlying pathologies producing the unusual clinical presentation of acute abdomen in dengue illness, which sometimes mimics acute appendicitis. Other possible causes of acute abdomen are the pathophysiologic changes that occur at the onset of shock in DHF or upon the simultaneous occurrence of acute appendicitis. To the best of our knowledge, vasculitis has not been reported in conjunction with dengue infection. The incidence of acute abdomen in dengue illness has been reported to be as high as $12 \%$, which occurred during a dengue epidemic in Pakistan [6]. Five (1.69\%) of the seven patients diagnosed with acute appendicitis in the report by Shamim underwent appendectomies, but histological confirmation was not mentioned [6]. It is important that clinicians in dengue-endemic areas be aware of this overlapping presentation in order to avoid unnecessary surgery-related morbidity or even mortality.

The patient we describe in this report developed acute appendicitis and dengue fever simultaneously. The development of an appendicular mass in our patient indicates a delay in diagnosis. Abdominal symptoms and signs were present upon his admission to our hospital, but they were misinterpreted as mimicry. Retrospectively, the persistence of the boy's fever beyond the viremic phase and the occurrence of neutrophil leukocytosis and raised serum CRP were indicators of the clinical diagnosis of dengue and acute appendicitis and supported the presence of an inflammatory process.

No test to measure our patient's erythrocyte sedimentation rate (ESR) was performed. It is a simple investigation that would have been helpful because ESR is normal in patients with DHF and lower than normal in those with shock [7], but is elevated in patients with acute appendicitis.

The case of our patient underscores the need for careful evaluation, including ultrasonography, of patients with acute abdomen, even when the diagnosis of dengue infection is confirmed. Delay in the diagnosis of dengue infection can cause dengue shock syndrome or even death. Likewise, delaying or missing the diagnosis of acute appendicitis can result in serious complications. This message is particularly pertinent during dengue seasons, when hospital admission rates to pediatric wards are very high.

\section{Consent}

Written informed consent was obtained from the patient's parents for publication of this case report and any accompanying images. A copy of the written consent is available for review by the Editor-in-Chief of this journal.

\section{Competing interests}

The authors declare that they have no competing interests.

\section{Authors' contributions}

MPS analyzed and interpreted the patient data and was the major contributor to the writing of the manuscript. MS provided his opinion regarding and confirmation of the diagnosis from a surgeon's point of view and agreed with the conservative management of the patient. The clinical care of the patient was shared by MS and MPS. Both authors read and approved the final manuscript.

\section{Acknowledgements}

We acknowledge all medical and nursing staff who helped in caring for our patient, as well as Dr Sankha Jayasinghe, Research Assistant in the Department of Paediatrics at the University of Colombo, for his help in preparing the manuscript. 


\section{Author details}

'Department of Pediatrics, Faculty of Medicine, University of Colombo, Kynsey Road, Colombo, Sri Lanka. 'Lady Ridgeway Hospital for Children, Colombo, Sri Lanka. ${ }^{3}$ Department of Surgery, Faculty of Medicine, University of Colombo, Kynsey Road, Colombo, Sri Lanka.

Received: 7 October 2013 Accepted: 5 February 2014

Published: 3 April 2014

\section{References}

1. McFarlane MEC, Plummer JM, Leake PA, Powell L, Chand V, Chung S, Tulloch K: Dengue fever mimicking acute appendicitis: a case report. Int J Surg Case Rep 2013, 4:1032-1034.

2. Premaratna R, Bailey MS, Ratnasena BG, de Silva HJ: Dengue fever mimicking acute appendicitis. Trans R Soc Trop Med Hyg 2007, 101:683-685.

3. Khor BS, Liu JW, Lee IK, Yang KD: Dengue hemorrhagic fever patients with acute abdomen: clinical experience of 14 cases. Am J Trop Med Hyg 2006, 74:901-904.

4. Khanna S, Vij JC, Kumar A, Singal D, Tandon R: Dengue fever is a differential diagnosis in patients with fever and abdominal pain in an endemic area. Ann Trop Med Parasitol 2004, 98:757-760.

5. Al-Araimi H, Al-Jabri A, Mehmoud A, Al-Abri S: Dengue haemorrhagic fever presenting as acute abdomen. Sultan Qaboos Univ Med J 2011, 11:265-268

6. Shamim M: Frequency, pattern and management of acute abdomen in dengue fever in Karachi, Pakistan. Asian J Surg 2010, 33:107-113.

7. Kalyanarooj S, Nimmannitya S: A study of erythrocyte sedimentation rate in dengue hemorrhagic fever. Southeast Asian J Trop Med Public Health 1989, 20:325-330.

doi:10.1186/1752-1947-8-116

Cite this article as: Senanayake and Samarasinghe: Acute appendicitis complicated by mass formation occurring simultaneously with serologically proven dengue fever: a case report. Journal of Medical Case Reports 2014 8:116.

\section{Submit your next manuscript to BioMed Central and take full advantage of:}

- Convenient online submission

- Thorough peer review

- No space constraints or color figure charges

- Immediate publication on acceptance

- Inclusion in PubMed, CAS, Scopus and Google Scholar

- Research which is freely available for redistribution 